\title{
La influencia de la escolarización en enseñanza media en la subjetividad sobre si mismos de los jóvenes que fracasan en ella.
}

\author{
Dr.Jesús M. Redondo
}

\begin{abstract}
Resumen
Se realiza un análisis sobre datos referidos a diversos aspectos de la percepción que sobre si mimos los adolescentes tienen al inicio y al término de su escolarización en el ciclo 14-16 de la enseñanza media en una muestra del País Vasco. Estos análisis nos permiten afirmar que la prolongación de la escolaridad con el actual diseño académico perjudica a los jóvenes que fracasan en la escuela, especialmente en aspectos de la subjetividad referidos a ellos mismos. Este perjuicio transforma diferencias personales en desigualdades sociales que se aceptan por efecto de la escolarización.
\end{abstract}

\section{Abstract}

It is carried an analysis of date referred to diverse aspect of the self perception that adolescents have in the beginning and end of their high school period, at the ages of 14 though 16 years, in a sample from the Vasco Country. These analysis allows us to sustain that the extension of the school period with the actual academic design, harms the youths that fail in school, especially in aspects of subjectivity related to themselves as students. This disadvantage transforms personal differences in social inequalities accepted by themselves as a result of school process.

\section{Introducción}

La presente investigación corresponde a un estudio sobre la influencia que la escolarización en la enseñanza media ha tenido sobre los jóvenes que se escolarizan en ella; comparando la influencia en los que tienen un buen desempeñoy aprueban, con la de los que reprueban.

La muestra con la que contamos es la misma que la señalada en una investigación anterior. La muestra es de 3.236 alumnos de EE.MM., mayoritariamente de REM (Programa experimental de reforma de la Enseñanza Media) (Villay otros,
1990, 22-24); pero con la ausencia de los que han fracasado en el primer ciclo de la enseñanza media por abandono o por repetición. Es decir, los que tienen éxito son los mismos, pero los que fracasan sólo son aquellos que han reprobado en segundo curso $(\mathrm{N}=2.515)$.

\begin{tabular}{|lllll|}
\hline \multicolumn{5}{|c|}{ Cuadro 0- } \\
Distribución dela Muestra \\
Abandono & 587 & $18,2 \%$ & & \\
Repetición & 134 & $4,1 \%$ & Fracaso & $\mathbf{4 7 , 7} \%$ \\
Reprobados & $\mathbf{8 2 2}$ & $25,4 \%$ & & \\
Aprobados & $\mathbf{1 6 9 3}$ & $52,3 \%$ & Exito & $\mathbf{5 2 , 3} \%$ \\
Total & 3236 & $100 \%$ & $100 \%$ & \\
\hline
\end{tabular}


Las variables que analizamos son algunas de las utilizadas para el contraste en la evaluación de la REM del País Vasco (Villa y otros, 1990, 24-26):

\section{a)Características sociológicas:}

* Edad.

*Sexo.

* Clase social del cabeza de familia.

\section{b)Características de historiaescolar:}

* Historial escolar en EGB.

*Exito-fracaso.

\section{c)Características personales:}

* Cat (CI).

* Vtea (aptitud verbal).

* Ctea (aptitud numérica).

* Hábitos de estudio (H).

* Dat (aptitud de razonamiento mecánico).

* Autoconcepto general (autoconcepto personal, social y escolar).

* Clima escolar (contexto interpersonal, contexto instructivo-imaginativo y contexto regulativo).

* Dem (actitudes democráticas y confianza en las instituciones).

* Satisfacción de los padres (percepción por el alumno de la) respecto a los estudios.

* Consideración del alumno por la familia (percepción por el alumno de la).

Las características técnicas de todas estas variables pueden encontrarse en el Anexo $1 \mathrm{de}$ "Rem" (Villa y otros, 1990, 211 y ss).

Aqui el fracaso lo entendemos operativamente como el conformado por el indicador: reprobado. Por ello las conclusiones que podemos sacar son referidas a las diferencias entre los aprobados y los reprobados; ya que carecemos de información para analizar la influencia de la escolarización en los que han abandonado o han repetido en primer curso de la EE.MM. De todas formas, podemos convenir que la influencia de la escolarización sobre estos jóvenes podria ser semejante a la que realiza la escolarización con los que son reprobados, pero quizás en mayor medida.

La pregunta que nos proponemos abordar en esta investigación, podriamos formularla como: Los que fracasan (son reprobados) en el primer ciclo de EE.MM.- que corresponde a la edad comprendida entre los 14 y 16 años -, ¿han sido favorecidos o desfavorecidos ensuscapacidadesintelectuales, ensus actitudes y en sus hábitos de estudio, durante su escolarizaciónen esteciclodela enseñanzamedia?.Entendiendo que de esta forma abordamos aspectos de la subjetividad de los jóvenes, especificamente referidos a la percepción de si mismos; ya que en esta etapa de la vida es esta autopercepción la que va configurando la propia identidad de forma muy significativa. Es desde esta última consideración que nos parece relevante el tema que nos proponemos investigar; ya que es la prolongación de la escolaridad en esta edad uno de los factores contextuales más determinante de la propia subjetividad de los jóvenes y, por tanto, conviene analizar críticamente si los resultados conseguidos se relacionan con los objetivos explícitos en las politicas públicas de educación.

Nuestra hipótesis de trabajo, en función del planteamiento que hemos expuesto a lo largo de nuestra reflexión en otras oportunidades, es que los alumnos "fracasados" han resultado desfavorecidos a lo largo de su escolarización en las actitudes y en los hábitos de estudio, aunque quizás no en las capacidades intelectuales.

Para comprobar estas hipótesis nos proponemos analizar :

1. Las diferencias de puntuación PRE (al inicio del ciclo) / POS (al final del ciclo), en las variables de capacidades intelectuales, actitudes hacia si mismo y hacia lo escolar, para los grupos de éxito/fracaso, mediante Razón t . 
2. Las diferencias PRE/POS para los grupos éxito/fracaso, mediante anova de medidas repetidas y neutralización de la situación inicial. Esta situación incial es la descrita a través de la puntuación en cinco factores PRE y en el historial escolar (HE).

3. Análisis factorial POS para comprobar la estabilidad de los factores hallados con los datos iniciales (PRE: al inicio de la enseñanza media) al realizarlo sobre los datos finales.

4. Análisis de varianza de los factores POS para los grupos de éxito/fracaso. Y análisis de varianza de los factores $\mathrm{POS}$ para los grupos de éxito/fracaso y sexo.

5. Diferencia PRE/POS mediante anova de medidas repetidas para los grupos de éxito/fracaso con neutralización de la situación incial.
Esta situación inicial la consideramos recogida en el historial escolar (HE) y en el sexo.

\section{Diferencia pre/ pos en los valores de las va riables segun los grupos de exito/ fracaso.}

1.1. Diferencias medidas mediante razón $\mathrm{t}$.

Hemos analizado las diferencias en el "Pos" para los grupos de éxito/fracaso, mediante Razón t. Los resultados que se reflejan en el Cuadro I.

De el podemos concluir que:

1.- El efecto de la escolarización en el primer ciclo de EE.MM. sobre los jóvenes que fracasan en el mismo parece semejante a la de los que tienen éxito en:

\begin{tabular}{|c|c|c|}
\hline \multicolumn{3}{|c|}{$\begin{array}{c}\text { Cuadro I- } \\
\text { Diferencias significativas (razón t) PRE/ POS en las } \\
\text { variables paragrupos éxito/ fracasoen EE.MM. }\end{array}$} \\
\hline & $\begin{array}{l}\text { DiferenciaPRE/ POS } \\
\text { Grupo EXITO }\end{array}$ & $\begin{array}{c}\text { Diferencia PRE/ POS } \\
\text { Grupo FRACASO }\end{array}$ \\
\hline No hay diferenciaPRE/POS & $\begin{array}{l}\text { F1 AU - Autoconcepto personal } \\
\text { F2 AU - Autoconcepto social } \\
\text { AU - Autoconcepto general } \\
\text { DEM - Actitudes democráticas }\end{array}$ & $\begin{array}{l}\text { F1 AU } \\
\text { F2 AU } \\
\text { DEM }\end{array}$ \\
\hline $\begin{array}{l}\text { Diferencia PRE/ POS } \\
\text { (disminución) }\end{array}$ & $\begin{array}{l}\text { SAF - Satisfacción familiar } \\
\text { CF - Consideración familiar } \\
\text { H - Hábitos de estudio } \\
\text { F3 AU - Autoconcepto escolar } \\
\text { Clima Educativo (F1, F2, F3) } \\
\text { GAE - Actitud ante el estudio }\end{array}$ & $\begin{array}{c}\text { SAF } \\
\text { CF } \\
\text { H } \\
\text { F3AU } \\
\text { F1, F2, F3 CL } \\
\text { GAE }\end{array}$ \\
\hline $\begin{array}{l}\text { DiferenciaPRE/ POS } \\
\text { (aumento) }\end{array}$ & $\begin{array}{l}\text { VTEA - Aptitud verbal } \\
\text { CTEA - Aptitud numérica } \\
\text { CAT - C. inteligencia } \\
\text { DAT - Razonamiento mecánico }\end{array}$ & $\begin{array}{l}\text { VTEA } \\
\text { CTEA } \\
\text { CAT } \\
\text { DAT }\end{array}$ \\
\hline
\end{tabular}


a) Estabilidad en:

Autoconcepto personal y social.

Actitudes democráticas.

b) Aumento en:

Aptitudes intelectuales.

Rendimiento académico.

c) Disminución en:

Satisfacción de la familia con los estudios.

Consideración del alumno por la familia como buen o mal estudiante.

Hábitos de estudio.

Clima educativo (F1CL,F2CL,F3CL ): clima interpersonal, instructivo, y regulativo percibido por los alumnos.

Autoconcepto escolar.

Actitud hacia el estudio.
2.- Losquefracasan en el primer ciclo deEnseñanza Media, además, la disminución del autoconcepto escolar va unida a una disminución en $\mathrm{d}$ Autoconceptogeneral.

1.2. Diferencia Pre/Pos para grupos (éxito/fracaso) mediante Anova de medidas repetidas y neutralización de la situación inicial.

En el apartado anterior señalábamos que "parece ..."; ya que para determinar si realmente la influencia de la escolarización en EE.MM. sobre aquellos que reprueban y los que tienen éxito es distinta y en qué, debemos neutralizar las situaciones diferentes de partida.

Las mejores variables, a nuestro entender, que neutralizan las situaciones de partida son:

El historial escolar (he).

La puntuación en los cinco factores encontrados en las variables iniciales (pre1, pre2, pre3, pre4 y pre5).

\begin{tabular}{|c|c|c|c|c|c|c|}
\hline & & $\begin{array}{r}\text { Analisis } \\
\text { variable } \\
\text { ttralización }\end{array}$ & $\begin{array}{l}\text { adro II.- } \\
\text { za PRE/ POS } \\
\text { dposéxito/ fra } \\
\text { iables desitu }\end{array}$ & $\begin{array}{l}\text { delas } \\
\text { caso. } \\
\text { ación in }\end{array}$ & & \\
\hline & Variable & RazónF & Significación & & Medias & \\
\hline & & (dif. fracas.) & & Grupo & PRE & POS \\
\hline F2 CL- & Climainstructivo- & 26,78 & $<001$ & Exito & 35.08 & 31.91 \\
\hline & imaginativo & & & Fracaso & 34.72 & 29.47 \\
\hline F3CL- & Clima regulativo & 13,95 & $<001$ & Exito & 17.94 & 17.11 \\
\hline & & & & Fracaso & 17.41 & 15.40 \\
\hline GAE - & Actitud hacia el & 97.17 & $<001$ & Exito & 8.71 & 8,86 \\
\hline & estudio & & & Fracaso & 9.10 & 9.96 \\
\hline F3 AU - & Autoconcepto & 52.57 & $<001$ & Exito & 13.18 & 12,78 \\
\hline & escolar & & & Fracaso & 11.42 & 10.18 \\
\hline $\mathrm{H}-$ & Hábitos de & 33,98 & $<001$ & Exito & 426.64 & 419.573 \\
\hline & estudio & & & Fracaso & 409.34 & 390.34 \\
\hline VTEA - & Aptitud verbal & 6.12 & $<05$ & Exito & 11.41 & 13,86 \\
\hline & & & & Fracaso & 10.79 & 12.79 \\
\hline
\end{tabular}


Hemos realizado un análisis de varianza (anovaSPSS) de medidas repetidas con neutralización de las variables señaladas como covariantes; y el resultado es el que se refleja en la Cuadro II. En el aparecen solamente las variables en las que el resultado es significativo.

De este Cuadro podemos deducir que:

1.- La escolarización en la EE.MM. comporta puntuaciones significativamente menores, tal como está diseñada y/o ejercida, para aquellos que fracasan respecto a los que tienen éxito en:

- $\quad$ Actitud hacia el estudio.

- $\quad$ El autoconcepto escolar o académico.

- Hábitos de estudio .

2.- Esto tiene su reflejo también, de alguna manera, en que estos jóvenes valoran menos (rechazan más) el clima escolar que se les ofrece en este ciclo de la enseñanza media tanto en su aspecto regulativo (F3CL) como en su aspecto instructivo-imaginativo (F2CL).

3.- Por último, aunque se podria considerar que también puntúan significativamente menos en su aptitud verbal, no lo tenemos en consideración al estar poco neutralizada su influencia en la situación inicial; ya que tiene poco peso en el factor tercero (F3 PRE), pues su comunalidad era de 0.28 .

Una conclusión general, quizás muy evidente, podria ser la necesidad de otro clima educativo, con más atención a la generación y consolidación de hábitos; y que tenga más en cuenta las propias actitudes y motivaciones de los jóvenes.

\section{Análisis factorial POS.}

Hemos realizado el análisis factorial de todas las variables ya señaladas en las notas del apartado introductorio; pero sobre sus valores en el POS; es decir, al finalizar el primer ciclo de la enseñanza

\begin{tabular}{|c|c|c|c|c|}
\hline \multicolumn{5}{|c|}{ Cuadro III.a.- } \\
\hline Variable & Comunalidad Factor & Autovalor & $\%$ de Var & $\% \operatorname{Var} A c u$ \\
\hline DF1AU & .67073 & 1 & 3.12757 & 19.519 .5 \\
\hline DF2AU & .72676 & 2 & 2.02691 & 12.732 .2 \\
\hline DF3AU & .64206 & 3 & 1.49050 & 9.341 .5 \\
\hline DCU14 & 63205 & 4 & 1.34405 & 8.449 .9 \\
\hline $\mathrm{DH}$ & .48796 & 5 & 1.03300 & 6.556 .4 \\
\hline DGAE & .38801 & | & & \\
\hline DDEM & .60232 & | & & \\
\hline DF1CL & .77147 & | & & \\
\hline DF2CL & .77046 & 1 & & \\
\hline DF3CL & .51027 & | & & \\
\hline DCAT & .52702 & 1 & & \\
\hline DDAT & .48748 & | & & \\
\hline DVTEA & .37168 & 1 & & \\
\hline DCTEA & .49246 & | & & \\
\hline $\mathrm{HE}$ & .44937 & | & & \\
\hline DCU24 & .49190 & | & & \\
\hline
\end{tabular}


media. El análisis factorial se realiza en iguales condiciones técnicas (SPSS, C.P., 5 factores, converge en 6 iteraciones, y Rotación Varimax). Las variables Pos presentan todas ellas una comunalidad aceptable, incluso la VTEA (aptitud verbal) que eleva su comunalidad en el Pos a 0.3716.

Los factores resultantes son semejantes, casi idénticos a los de las variables PRE; con la salvedad de que el primer factor cambia de sentido: "Aceptación de la escuela". Lo cual es debido fundamentalmente a la disminución de sujetos con rechazo en el POS, ya que no están incluidos ni los repetidores, ni los abandonos.

Otra salvedad está en el factor quinto que ahora tiene un autovalor=1.03, y adquieren en él un mayor peso las actitudes democráticas, sobrepasando a la clase social, por lo que es pertinente el nombre de "clase socio-cultural".

El total de la varianza explicada es de $56,4 \%$, prácticamente la misma que en factorial Pre. Todo esto se refleja en los Cuadros III. a y b.

\section{1. - Análisis de varianza de los factores pos para losgruposdeéxito/ fracaso.}

Sometemos los factores "pos" a un análisis de varianza para los grupos de éxito/fracaso y tenemos lo que se refleja en el Cuadro IV.

De él podemos resaltar:

1. El factor socio-cultural no discrimina para éxito/fracaso entre aquellos que llegan al final del primer ciclo de enseñanzas medias. Es decir, una vez exceptuados los abandonos y los repetidores.

2. Los factores más discriminantes siguen siendo el de aceptación/rechazo (factor primero), y el

\section{Cuadro III.b-}

\section{Análisis factorial pos. matriz factores rotados}

\section{$\begin{array}{lllll}\text { FACTOR } 1 & \text { FACTOR } 2 & \text { FACTOR } 3 & \text { FACTOR } 4 & \text { FACTOR } 5\end{array}$}

DF1AU

.12848

.01428

.12247

$798 / 3$

.03233

DF2AU

.02915

.05745

$-.03924$

84903

.01453

DF3AU

.72382

.19346

.09367

26818

.00508

DCU14

$-77602$

$-.05237$

DH

.6078

.16028

$-.15644$

$-.05099$

.00481

DGAE

$-60713$

$-.08243$

$-.01559$

30309

$-.02553$

.00615

.04335

.10342

DDEM

.05999

$-.03576$

.14919

.06871

.75529

DF1CL

.02659

.87568

.00038

.06235

.00784

DF2CL

.13405

.86164

$-.01386$

.08706

.04788

DF3CL

.18508

.68813

.01663

$-.03739$

$-.02863$

DCAT

.13451

.05155

71085

$-.02482$

.01879

DDAT

$-.00851$

.00841

.69370

.07538

$-.02103$

DVTEA

.06910

$-.05706$

59658

.02218

$-.08512$

DCTEA

.09360

.01425

.69390

$-.00419$

.04456

$\mathrm{HE}$

$-56209$

.01880

$-22579$

26016

$-.12007$

DCU24

$-11192$

.05556

$-.03396$

.6552 
Cuadro IV.-

\section{Análisis de varianza de los factores pos para el grupo éxito/ fracaso}

\begin{tabular}{|c|c|c|c|c|c|}
\hline & Variable & Razón F & Significación & \multicolumn{2}{|c|}{ Exito/Fracaso Medias de grupc } \\
\hline $\mathrm{Fl}$ & Aceptación & 685,16 & $<0001$ & éxito & .3432 \\
\hline POS & escolar & & & fracaso & -.7065 \\
\hline $\mathrm{F} 2$ & Clima educativo & 27.82 & $<0001$ & éxito & .0790 \\
\hline POS & & & & fracaso & -.1625 \\
\hline F3 & Aptitudes & 78,03 & $<0001$ & éxito & .9375 \\
\hline POS & intelectuales & & & fracaso & -.2691 \\
\hline F4 & Autoconceptos & 10.06 & $<005$ & éxito & -.0478 \\
\hline POS & relacional activo & & & fracaso & .0984 \\
\hline F5 & Clase socio- & 0000 & $>05$ & éxito & .0001 \\
\hline POS & cultural & & & fracaso & -.0001 \\
\hline
\end{tabular}

\begin{tabular}{|c|c|c|c|c|c|}
\hline \multicolumn{6}{|c|}{$\begin{array}{c}\text { Cuadro V.- } \\
\begin{array}{c}\text { Análisis doble de varianza (fracaso y sexo) } \\
\text { delosfactores pos }\end{array} \\
\end{array}$} \\
\hline \multicolumn{2}{|r|}{ Variables } & \multicolumn{2}{|c|}{ Razón } & \multirow{2}{*}{$\frac{\text { Sig. }}{<001}$} & \multirow[t]{2}{*}{ Grupos } \\
\hline POS 1 & Aceptación & $\mathrm{ME}$ & 399,98 & & \\
\hline & escolar & Fracaso & 692,21 & $<001$ & $1^{\circ}$ Chicas éxito \\
\hline & & Sexo & 89,18 & $<001$ & $2^{\circ}$ Chicos éxito \\
\hline & & Interacción & 1,09 & $>05$ & $3^{\circ}$ Chicas fracaso \\
\hline & & Total & 267,45 & $<001$ & $4^{\circ} \mathrm{Chicos}$ fracaso \\
\hline \multirow[t]{5}{*}{ POS2 } & Clima & $\mathrm{ME}$ & 22,91 & $<001$ & \\
\hline & & Fracaso & 26,79 & $<001$ & $1^{\circ}$ Chicas éxito \\
\hline & & Sexo & 17,45 & $<001$ & $2^{\circ}$ Chicos éxito \\
\hline & & Interacción & .67 & $>05$ & $3^{\circ} \mathrm{Chicas}$ fracaso \\
\hline & & Total & 15.50 & $<001$ & $4^{\circ} \mathrm{Chicos}$ fracaso \\
\hline \multirow[t]{5}{*}{ POS 3} & Aptitudes & $\mathrm{ME}$ & 97,39 & $<001$ & \\
\hline & Intelectuales & Fracaso & 88,82 & $<001$ & $1^{\circ}$ Chicos éxito \\
\hline & & Sexo & 112,80 & $<001$ & $2^{\circ}$ Chicos fracaso \\
\hline & & Interacción & .071 & $>05$ & $3^{\circ}$ Chicas éxito \\
\hline & & Total & 64.955 & $<001$ & $4^{\circ} \mathrm{Chicas}$ fracaso \\
\hline \multirow[t]{5}{*}{ POS 4} & Autoconcepto & $\mathrm{ME}$ & 27,71 & $<001$ & \\
\hline & relacional & Fracaso & 8,79 & $<001$ & $1^{\circ} \mathrm{Chicos}$ fracaso \\
\hline & activo & Sexo & 45,16 & $<001$ & $2^{\circ}$ Chicos éxito \\
\hline & & Interacción & .041 & $>05$ & $3^{\circ}$ Chicas fracaso \\
\hline & & Total & 18,49 & $<001$ & $4^{\circ}$ Chicas éxito \\
\hline \multirow[t]{5}{*}{ POS 5} & Case & $\mathrm{ME}$ & 237 & $>05$ & \\
\hline & Socio-cultural & Fracaso & .004 & $>05$ & $1^{\circ}$ Chicos fracaso \\
\hline & & Sexo & 4.71 & $<05$ & $2^{\circ}$ Chicas éxito \\
\hline & & Interacción & 7.63 & $<01$ & $3^{\circ}$ Chicos éxito \\
\hline & & Total & 4.11 & $<01$ & $4^{\circ} \mathrm{Chicas}$ fracaso \\
\hline
\end{tabular}


de aptitudes intelectuales (factor tercero); aún cuando siguen siendo también discriminadores el factor segundo (clima educativo) y el factor cuarto (autoconcepto relacional activo). En este último puntúan más los grupos de fracaso.

\subsection{Análisis de varianza de los factores pos para los grupos deéxito/ fracaso y sexo.}

Al realizar el análisis de varianza doble respecto a grupos de éxito/fracaso y sexo observamos lo que aparece en el Cuadro V. Del cual destacamos :

a) La puntuación en el factor primero (aceptación/rechazo escolar) está relacionada con el éxito/fracaso de forma muy claray mucho más que con el sexo.

b) La puntuación en el factor segundo (clima escolar) está relacionada con el éxito/fracaso más que con el sexo; cambiando lo que ocurria en el factor pre, que aparecia más relacionado con el sexo que con el éxito / fracaso. c) El factor tercero está más asociado con el sexo que con el éxito/fracaso ; cambiando también lo señalado en el factor Pre, en el que ocurria lo contrario.

d) El factor cuarto (autoconcepto relacional) está más asociado al sexo que al éxito/fracaso.

e) La clase socio-cultural (factor quinto) está poco asociado y al sexo.

Para interpretar estas variaciones, y sobre todo la del último factor, conviene analizar la población que llega al final del ciclo y su distribución de clase; comparándola con la de inicio del mismo. Esto lo podemos apreciar en el Cuadro VI.

En el apreciamos la significatividad de la mayor pérdida de efectivos de la clase baja y muy baja; éstas pérdidas las encontraremos luego recogidas en las características sociológicas de los jóvenes de la Iniciación Profesional (garantía social), programa creado como alternativa para los jóvenes que fracasan en la escuela y carecen de empleo.

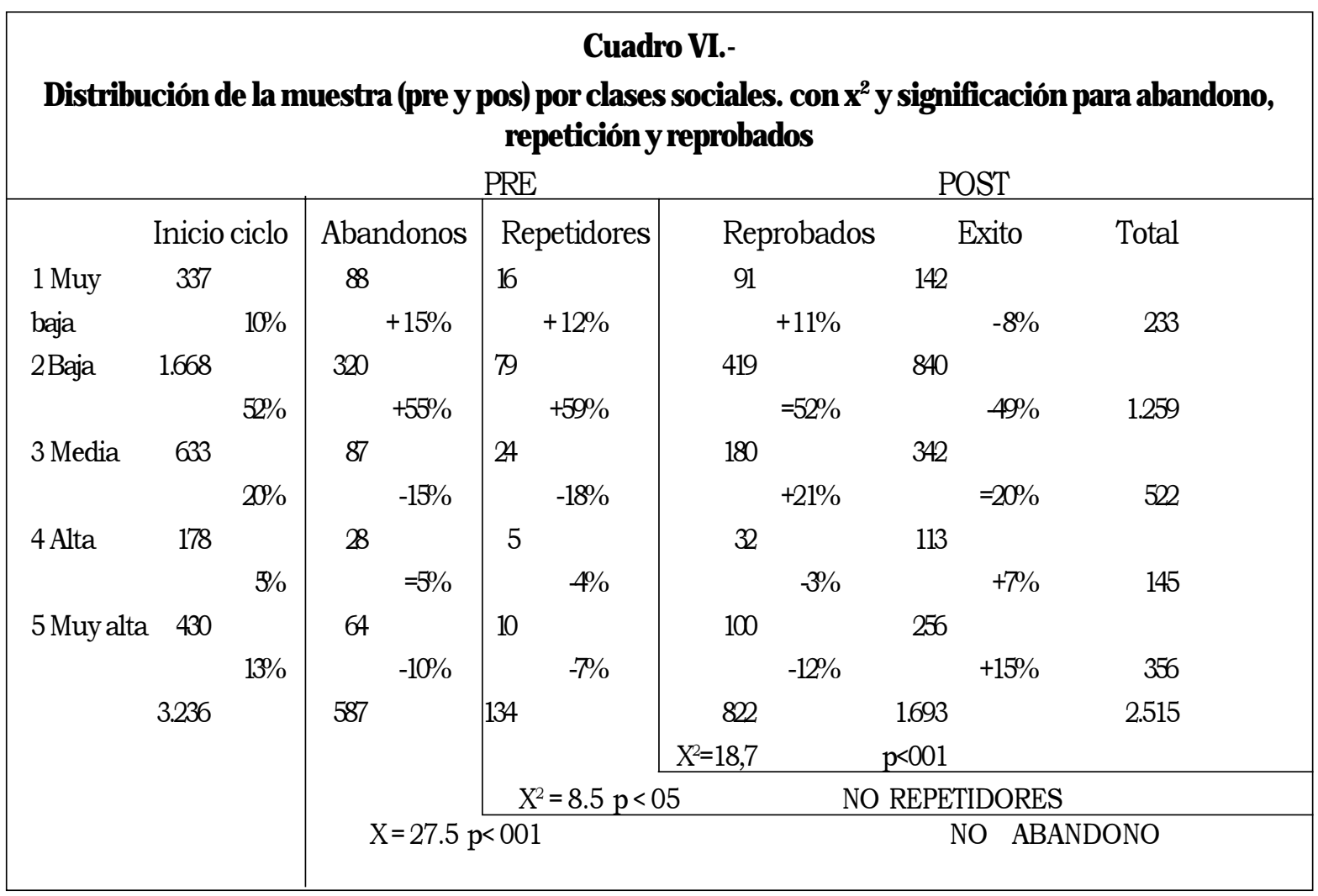




\subsection{Diferencias pre/ pos mediante anova de medidas repetidas y neutralización delasituación inicial.}

Hemos realizado también un análisis de las diferencias significativas entre los factores Pre y Pos para los grupos de éxito/fracaso; mediante análisis anova-Spss de medidas repetidas con neutralización del sexo y del historial escolar. Los resultados aparecen en el Cuadro VII.

\begin{tabular}{|c|c|c|}
\hline $\begin{array}{l}\text { Análisis de Vari } \\
\text { diferencia delos }\end{array}$ & $\begin{array}{l}\text { Cuadro VII.- } \\
\text { anza de medid } \\
\text { factores PRE/ } \\
\text { leexito/ fracaso }\end{array}$ & $\begin{array}{l}\text { repetidas de } \\
\text { OS paragrupos }\end{array}$ \\
\hline con neutraliz & zación deSexoe Histc & al Escolar \\
\hline FACTORES & F (FRAC/DIF) & Significación \\
\hline $\begin{array}{l}\text { Dif. PRE/POS F1 } \\
\text { Aceptacción/Recha }\end{array}$ & 254.11 & $<001$ \\
\hline $\begin{array}{l}\text { Dif. PRE/POS F2 } \\
\text { ClimaEducativo }\end{array}$ & 6.07 & $<05$ \\
\hline $\begin{array}{l}\text { Dif. PRE/POS F3 } \\
\text { Aptitudes Intelectu }\end{array}$ & ales & $>05$ \\
\hline $\begin{array}{l}\text { Dif. PRE/POS F4 } \\
\text { Autoconcepto R.A. }\end{array}$ & .58 & $>05$ \\
\hline $\begin{array}{l}\text { Dif. PRE/POS F5 } \\
\text { Clase Socio-Cultura }\end{array}$ & 1.07 & $>05$ \\
\hline
\end{tabular}

De éste cuadro podemos deducir:

a) Que la escolarización en el primer ciclo de EE.MM. parece resultar desfavorable (disminuyen las puntuaciones) a los jóvenes que fracasan (son reprobados), especialmente en su actitud de aceptación de la escuela (factor primero): autoconcepto escolar, consideración de la familia, hábitos de estudio, actitud hacia el estudio, etc. Es decir aspectos de su subjetividad referidos a la percepción sobre si mismos como alumnos.

b) Que la escolarización en el primer ciclo de la EE.MM. no tiene influencia significativa diferente para el factor tercero (aptitudes intelectuales), es decir, no resulta desfavorable a los alumnos que reprueban, sino que aumenta sus capacidades intelectuales en la misma proporción que los de éxito; aunque también podemos indicar que la escolarización en enseñanza media, ciertamente no actúa como compensación de las desigualdades de aptitudes intelectuales, " presuntamente naturales", en los jóvenes que fracasan en ella.

c) Que la escolarización en el primer ciclo de EE.MM. tampoco interviene de forma diferente para los que fracasan, en el autoconcepto relacional activo, ni, evidentemente, en la clase socio-cultural.

\section{Conclusiones}

1. Los jóvenes que fracasan (son reprobados) en el primer ciclo de EE.MM. no han sido desfavorecidos en el desarrollo de sus capacidades intelectuales; sino por el contrario las han aumentado en la misma proporción que lo han hecho los que tienen éxito. Esto quiere decir que, como partían con puntuaciones menores, siguen manteniendo comparativamente las mismas diferencias con los de éxito que al principio del ciclo. La escolarización no ha "compensado"las diferencias de aptitudes intelectuales.

2. Los jóvenes que fracasan (son reprobados) resultan significativamente desfavorecidos en sus hábitos de estudio, en sus actitudes hacia el estudio, y en sus actitudes hacia si mismos respecto a los estudios (autoconcepto escolar). Todos estos aspectos se agrupan en el Factor 1 : Rechazo / Aceptación de la escuela. Es decir, que al aumentar su rechazo escolar la escolarización consolida la "inferioridad" de los fracasados; no sólo, ni tanto, por sus menores puntuaciones en las capacidades intelectuales; sino por la disminución significativamente mayor de sus actitudes hacia si mismos y sus hábitos de estudio; es decir en aspectos que construyen y configuran su subjetividad referida a si mismos como alumnos. La escolarización parece haber transformado las 
diferencias individuales de aptitudes, en desigualdades sociales sancionadas externamente (fracaso escolar, no consecución del título), y asumidas (internamente) por el propio individuo en sus actitudes hacia si mimos y en sus hábitos de estudio (aspectos de su subjetividad).

3. La afirmación anterior se manifiesta, de otra forma, en que los que fracasan presentan una diferencia significativa de puntuaciones en el factor de clima escolar (PRE/POS); específicamente, debido a la disminución mayor de la valoración del clima instructivo-imaginativo y del clima regulativo. Estas diferencias vienen a reiterar un aumento del rechazo del clima escolar en los que reprueban, por efecto de la escolarización.

4. El fracaso que operativamente hemos analizado como conformado por el indicador: reprobados; se nos manifiesta, tras los análisis realizados, como un fracaso que se transforma de externo (ser reprobado), en interno (actitudes y hábitos respecto al estudio). Este fracaso es debido, por una parte, a que responde a una evaluación referida a normas, es decir a someter a todos los alumnos a un estandar general; y no estar, la evaluación, realizada en base a criterios personalizados o individualizados (evaluación criterial). Por otra parte, desde un punto de vista más social, hay autores, y concretamente Perronoud (1990) que indican que podemos considerar este fracaso como "injusto", al no valorar el aumento de capacidades o rendimientos de cada sujeto, sino que someter a una comparación con normas de excelencia fabricadas por el mismo sistema escolar y que responden a intereses de grupos sociales específicos.

De todas formas, en este análisis no hemos podido considerar al colectivo de jóvenes que abandonan o repiten. Resultaria de un gran interés que alguna investigación realizara un seguimiento y análisis sobre las características de estos jóvenes y sobre los efectos que la escolarización normalizada $\mathrm{y} /$ o mediante otros programas socioeducativos tie- ne sobre ellos, descubriendo con más precisión la influencia favorable o desfavorable de la prolongación de la escolaridad en sus diversas modalidades. Si hemos podido percibir, en cambio, que eran sujetos en mayor proporción procedentes de las clases bajas.

Por otra parte, también nos quedan por considerar los jóvenes que no llegan a escolarizarse en la enseñanza media, sino que abandonan su escolarización en la EGBy se incorporan a programas de capacitación laboral; en el caso del País Vasco al programa de Iniciación Profesional.

\section{Bibliografia}

Fernández 0. (1988) "Comprensión y manejo del análisis factoria". REVISTA INTERNACIONAL DE SOCIOLOGİA; 46 (1), 7-35. Centro Superior de Investigaciones Cientificas. Madrid.

RedondoJ .M. (1995) "Análisis de la situación social de los jóvenes desfavorecidos ypropuestade un programaintegral de garantía social". Tesis doctoral. Universidad de Deusto. Edición microficha.

RedondoJ .M. (1997)"La dinámica escolar: de la diferencia a la desigualdad" REVISTA DE PSICOLOGÍA. Universidad de Chile. Vol VI. 7-18. Santiago de Chile.

RedondoJ .M. y Otros. (1998) "El mundo de losjóvenes yla reforma de la enseñanza media" REVISTA DE PSICOLOGÍA. Universidad de Chile. Vol VII. 35-49. Santiago de Chile.

RedondoJ .M. (1998). "Características psicosocioeducativas de los alumnos que fracasan en el primerciclo de la EE.MM. en el País Vasco". Revista Enfoques Educacionales. Vol 1. № 2. 117-139. Universidad de Chile.

VillaA. YOtros. (1990) "REM: Reforma de las Enseñanzas Medias. Evaluación del Primer Ciclo del Plan Experimental de la Comunidad Autónoma Vasca". Vitoria: Gobierno Vasco. 